\title{
Publisher Correction: Exposure to Stress-Dose Steroids and Lethal Septic Shock After In-Hospital Cardiac Arrest: Individual Patient Data Reanalysis of Two Prior Randomized Clinical Trials that Evaluated the Vasopressin-Steroids-Epinephrine Combination Versus Epinephrine Alone
}

\author{
Spyros D. Mentzelopoulos ${ }^{1,2}$ (D) $\cdot$ losifina Koliantzaki ${ }^{1,3}$ - Marios Karvouniaris ${ }^{4}$. Charikleia Vrettou ${ }^{1}$. \\ Nicolas Mongardon ${ }^{5,6,7} \cdot$ George Karlis ${ }^{1}$. Demosthenes Makris ${ }^{4}$. Epaminondas Zakynthinos ${ }^{4} \cdot$ Sotirios Sourlas $^{8}$. \\ Stavros Aloizos ${ }^{9} \cdot$ Theodoros Xanthos $^{8} \cdot$ Spyros G. Zakynthinos $^{1}$
}

Published online: 19 September 2018

(C) Springer Science+Business Media, LLC, part of Springer Nature 2018

Publisher Correction: Cardiovascular Drugs and Therapy (2018) 32:339-351

https://doi.org/10.1007/s10557-018-6811-0

The original version of this article unfortunately contained the following mistakes.

Assignment of author affiliations for Sotirios Sourlas, Stavros Aloizos and Theodoros Xanthos were incorrect.They are now corrected in this erratum.

In Table 2, the frequency of Septic Shock reported just below the frequency of "At least 1 Episode of VAP" actually corresponds to the First (and not the Second) Episode of VAP during the postresuscitation period. The corrected Table is shown next page.

The online version of the original article can be found at https://oi.org/ 10.1007/s10557-018-6811-0

Spyros D. Mentzelopoulos

sdmentzelopoulos@yahoo.com; sdmentzelopoulos@gmail.com; sdm@hol.gr

1 First Department of Intensive Care Medicine, University of Athens Medical School, Athens, Greece

2 Department of Intensive Care Medicine, Evaggelismos General Hospital, 45-47 Ipsilandou Street, GR-10675 Athens, Greece

3 Department of Anesthesiology, Attikon University Hospital, Athens, Greece

4 Department of Intensive Care Medicine, University of Thessaly Medical School, Larissa, Greece
5 Service d'Anesthésie et des Réanimations Chirurgicales, Réanimation Chirurgicale Cardio-vasculaire DHU A-TVB, Hôpitaux Universitaires Henri Mondor, Assistance Publique des Hôpitaux de Paris, Créteil, France

6 Faculté de Médecine, Université Paris Est Créteil, Créteil, France

Equipe 3 «Stratégies Pharmacologiques et Thérapeutiques Expérimentales des Insuffisances Cardiaques et Coronaires», INSERM U955, Créteil, France

8 School of Medicine, European University Cyprus, Nicosia, Cyprus

9 Department of Intensive Care Medicine, 401 Greek Army Hospital, Athens, Greece 
Table 2 Postresuscitation infectious complications

Type of Infection and Pathogens

VAP 1st episode ${ }^{\mathrm{a}}$ - pathogen, $n(\%)$
At least 1 episode of VAP, $n(\%)$
Septic shock during VAP 1 st Episode, $n(\%)$
VAP 2nd episode ${ }^{\mathrm{a}}$ - pathogen, $n(\%)$

At least 2 episodes of VAP, $n(\%)$

Septic shock during VAP 2 nd episode, $n(\%)$

VAP 3rd episode ${ }^{\mathrm{a}}$ - pathogen, $n(\%)$

3 episodes of VAP, $n(\%)$

Septic shock during VAP 3rd episode, $n(\%)$

Type of Infection and Pathogens

VAT $^{\mathrm{a}}$ - pathogen, $n(\%)$

VAT, $n(\%)$

Septic shock during VAT, $n(\%)$

Lethal septic shock - pathogen, $n(\%)$

Lethal septic shock, $n$ (\%)

Lethal septic shock in survivors for $\geq 48 \mathrm{~h}, \mathrm{n} / \mathrm{total} N$ (\%)

Catheter-related bacteremia - pathogen, $n(\%)$

Catheter-related bacteremia, $n(\%)$

Septic Shock during catheter-related bacteremia, $n(\%)$

Urinary tract infection - pathogen, $n(\%)$

Urinary tract infection, $n(\%)$

Septic shock during urinary tract infection, $n(\%)$

Other complication - Endocarditis - pathogen, $n(\%)$

Transfusion-related

Soft tissue infection

Other infectious complication, $n(\%)$

Septic shock during other infectious complication, $n(\%)$

Antibiotic Resistance ${ }^{\mathbf{x}}$

Infection due to XDR pathogen, $n(\%)$

Infection due to MDR pathogen, $n(\%)$

Infection due to non-MDR pathogen, $n(\%)$

Infection due to fungus, $n(\%)$
Steroids

No Steroids

$(n=118)$

$(n=73)$

$p$ value

A. baumannii $^{\mathrm{b}}$
K. pneumoniae $\mathrm{c, \textrm {d }}$
P. aeruginosa
MRSA
Pr. mirabilis
E. aerogenes

$18(15.3)$

$12(10.2)$

$5(6.9)$

$10(8.5)$

$1(0.8)$

$1(0.8)$

$0(0.0)$

42 (35.6)

$34(28.8)$

P. aeruginosa

$K$. pneumoniae

$5(4.2)$

A. baumannii

Prov. Stuartii

K. pneumoniae

$3(2.5)$

2 (1.7)

$1(0.8)$

$11(9.3)$

$4(5.5)$

$2(1.7)$

2 (1.7)

$1(0.8)$

Steroids

$(n=118)$

A. baumannii ${ }^{\mathrm{f}}$

K. pneumoniae $^{\mathrm{g}}$

$P$. aeruginosa

S. maltophilia ${ }^{\mathrm{h}}$

2 (1.7)

$0(0.0)$

$0(0.0)$

$1(0.8)$

$3(2.5)$

2 (1.7)

K. pneumoniae ${ }^{\mathrm{i}}$

A. baumannii ${ }^{k}$

P. aeruginosa ${ }^{l}$

Pr. mirabilis ${ }^{\mathrm{m}}$

Prov. stuartii ${ }^{\mathrm{n}}$

E. aerogenes ${ }^{\circ}$

R. Radiobacter $^{\mathrm{p}}$

Str. pyogenes ${ }^{\mathrm{q}}$

C. glabrata

C. parapsilosis ${ }^{\mathrm{s}}$

T. asachii ${ }^{\mathrm{t}}$

Prov. stuartii

$K$. pneumoniae

P. aeruginosa

C. parapsilosis

P. aeruginosa

A. baumannii

E. aerogenes

Pr. mirabilis

C. tropicalis

K. pneumaniae

A. baumannii

$C M V^{\mathrm{u}}$

Str. pyogenes ${ }^{\mathrm{v}}$

$11(9.3)$

$3(2.5)$

$2(1.7)$

$0(0.0)$

$0(0.0)$

$0(0.0)$

$1(0.8)$

$1(0.8)$

$1(0.8)$

$0(0.0)$

$1(0.8)$

20 (16.9)

$20 / 83(24.1)$

$2(1.7)$

$0(0.0)$

$1(0.9)$

$1(0.9)$

4 (3.4)

$4(3.4)$

$2(1.7)$

$0(0.0)$

$0(0.0)$

$1(0.8)$

$1(0.8)$

$4(3.4)$

$1(0.8)$

$1(0.8)$

$1(0.8)$

$1(0.8)$

$1(0.8)$

4 (3.4)

4 (3.4)

Steroids

$\left(\mathrm{n}_{\mathrm{I}}=82\right)^{\mathrm{y}}$

$50(61.0)$

$23(28.0)$

$6(7.3)$

$3(3.7)$
$8(11.0)$

$2(2.7)$

$2(2.7)$

$1(1.4)$

$1(1.4)$

$9(26.0)$

$15(20.5)$

0.20

3 (4.1)

1 (1.4)

$0(0.0)$

$0(0.0)$

$4(5.5)$

7 (5.9)

1 (1.4)

1 (1.4)

1 (1.4)

0.42

$>0.99$

$>0.99$

$>0.99$

No Steroids

$(n=73)$

$0(0.0)$

1 (1.4)

1 (1.4)

$0(0.0)$

2 (2.7)

2 (2.7)

10 (13.7)

1 (1.4)

1 (1.4)

$1(1.4)$

$1(1.4)$

$1(1.4)$

$0(0.0)$

$0(0.0)$

$0(0.0)$

$1(1.4)$

$0(0.0)$

16 (21.9)

$16 / 37$ (43.2)

1 (1.4)

1 (1.4)

$0(0.0)$

$0(0.0)$

$2(2.7)$

$2(2.7)$

1 (1.4)

1 (1.4)

1 (1.4)

$0(0.0)$

$0(0.0)$

3 (4.1)

$2(2.7)$

1 (1.4)

$0(0.0)$

$0(0.0)$

$0(0.0)$

1 (1.4)

1 (1.4)

No Steroids

$\left(\mathrm{n}_{\mathrm{I}}=46\right)^{\mathrm{y}}$

$31(67.4)$

$13(28.3)$

1 (2.2)

1 (2.2)
$>0.99$

0.64

0.45

0.051

$p$ value

$>0.99$

$>0.99$

$>0.99$

0.60

0.65

0.65

$p$ value

0.57

$>0.99$

0.42

$>0.99$ 
Summary data pertaining to the primary outcome are highlighted in italic bold script.

SOFA, sequential organ failure assessment; VAP, ventilator-associated pneumonia; VAT, ventilator-associated tracheobronchitis; A., Acintobacter; K., Klebsiella; P. Pseudomonas; MRSA, Methicillin-resistant Staphylococcus aureus; Pr. Proteus; E. Enterobacter; Prov. Providenzia; S. Stenotrophomonas; R. Rhizobium; Str. Streptococcus; C, Candida; T. Trichosporon; CMV, Cytomegalovirus; XDR, extensively drug-resistant; MDR, multi drug-resistant.

${ }^{\text {a }}$ VAP, first episode was caused by multidrug-resistant (MDR) /extensively drug-resistant (XDR) bacteria in 16/25 patients of the Steroids group and 3/16 patients of the No Steroids group; in addition, there was 1 case of VAP by a non-MDR bacterium in each group; VAP second episode was caused by MDR/XDR bacteria in 3/8 patients of the Steroids group and 2/2 patients of the No Steroids group; VAP third episode was caused by XDR bacteria in 2 patients of the Steroids group and 1 patient of the No Steroids group; VAT was caused by MDR/XDR bacteria in 0/3 patients of the Steroids group and 1/1 patient of the No Steroids group

b Temporally associated with (i.e., diagnosis established within $72 \mathrm{~h}$ prior to) acute respiratory distress syndrome (ARDS) in 2 patients of the Steroids group and 1 patient of the No Steroids group

c Temporally associated with ARDS in 8 patients of the Steroids group and 4 patients of the No Steroids group

${ }^{\mathrm{d}}$ VAP occurred in 1 patient of the No Steroids group on day 1 postarrest; the patient was transferred intubated and mechanically ventilated from another hospital before the cardiac arrest and the total time on mechanical ventilation before VAP diagnosis was 4 days

e Temporally associated with ARDS in 4 patients of the Steroids group and 1 patient of the No Steroids group

${ }^{\mathrm{f}}$ Temporally associated with ARDS in 2 patients of the Steroids group

gemporally associated with ARDS in 1 patient of the No Steroids group

h Temporally associated with ARDS in 1 patient of the Steroids group

${ }^{\mathrm{i}}$ Cultures of blood isolates revealed XDR K. Pneumoniae in 20 cases of both groups and MDR K. Pneumoniae in 1 case of the No Steroids group; septic shock causing death occurred within 7 days of VAP onset by an XDR and/or an MDR pathogen in 10 patients of the Steroids group (with 6 patients also developing ARDS) and 4 patients of the No Steroids group (with 2 patients also developing ARDS); in 1 patient of the No Steroids group, lethal septic shock occurred within 1 day of onset of VAT by XDR K. Pneumoniae; in 1 patient of the Steroids group, lethal septic shock occurred in the presence of clinical suspicion of acalculus cholecystitis; in 4 patients of the No Steroids group, lethal septic shock occurred as a complication of intraabdominal sepsis ( 2 cases of postoperative abscess formation, 1 case of Crohn's disease-related abscess formation, and 1 case of suspected acalculus cholecystitis); in 1 patient of the No Steroids group, lethal septic shock occurred as a complication of catheter-related infection by XDR K. Pneumoniae

${ }^{\mathrm{k}}$ Cultures of blood isolates revealed XDR A. Baumannii in 3 cases (No Steroids group, $n=1$ ), and MDR A. Baumannii in 1 case; septic shock causing death occurred within 5 days of VAP onset by an XDR and/or an MDR pathogen in 3 patients of the Steroids group (with 1 patient also developing ARDS); in 1 patient of the No Steroids group, lethal septic shock due to presumed cholangitis occurred within $48 \mathrm{~h}$ of endoscopic retrograde cholangiopancreatography for pre-arrest-confirmed choledocholithiasis

${ }^{1}$ Cultures of blood isolates revealed XDR P. Aeruginosa in 2 cases (No Steroids group, $\mathrm{n}=1$ ), and MDR P. Aeruginosa in 1 case; septic shock causing death occurred within 1 day of VAP onset by an XDR and/or an MDR pathogen in 2 patients of the Steroids group (with both also developing ARDS); in 1 patient of the No Steroids group

${ }^{\mathrm{m}}$ Cultures of blood isolates revealed MDR Pr. Mirabilis in 1 patient of the No Steroids group; septic shock causing death occurred within 4 days of VAP onset by XDR E. Aerogenes

${ }^{\mathrm{n}}$ Cultures of blood isolates revealed MDR Prov. Stuartii in 1 patient of the No Steroids group; septic shock causing death occurred within 5 days of onset of urinary tract infection by MDR E. Aerogenes

${ }^{\circ}$ Cultures of blood isolates revealed MDR E. Aerogenes in 1 patient of the No Steroids group; septic shock causing death occurred within 3 days of onset of VAP by MDR MRSA

${ }^{\mathrm{p}}$ Cultures of blood isolates revealed non-MDR R. Radiobacter in 1 patient of the Steroids group; septic shock causing death and ARDS occurred within 1 day of onset of VAP by XDR A. Baumannii

${ }^{\mathrm{q}}$ Cultures of blood isolates revealed non-MDR Str. Pyogenes; septic shock causing death occurred within 4 days of onset of soft tissue infection by the aforementioned pathogen

${ }^{\mathrm{r}}$ Cultures of blood isolates revealed fluconazole-resistant $C$. Glabrata; septic shock causing death occurred within 2 days of onset of peritonitis that followed ERCP for choledocholithiasis - cholelithiasis was already confirmed during pre-arrest hospital stay

${ }^{s}$ Cultures of blood isolates revealed fluconazole-resistant C. Parapsilosis; septic shock causing death and ARDS occurred within 2 days of onset of peritonitis secondary to suspected postenterectomy anastomosis leakage

${ }^{\mathrm{t}}$ Cultures of blood isolates revealed Amphotericin B and voriconazole-susceptible T. Asachii causing poststernotomy sternal osteomyelitis and mediastinitis and lethal septic shock

u Associated with severe bilateral pneumonia and ARDS

${ }^{v}$ Associated with lethal septic shock by the same pathogen

${ }^{x}$ Definitions provided in the eMethods section of the Supplement

${ }^{y} n_{I}$, reflects number of episodes of postresuscitation infections that occurred in 48 and 25 patients of the Steroids and No Steroids group, respectively 